\title{
重费米子材料 $\mathrm{CeCoIn}_{5}$ 超导态的唯象理论
}

李宇 ${ }^{1,2}$, 杨义峰 $1,2,3^{*}$

1. 中国科学院物理研究所, 北京凝聚态物理国家实验室, 北京 100190 ;

2. 中国科学院大学, 北京 100049 ;

3. 量子物质科学协同创新中心, 北京 100190

* 联系人, E-mail: yifeng@iphy.ac.cn

2017-06-22 收稿, 2017-08-02 修回, 2017-08-04 接受, 2017-11-16 网络版发表 国家优秀青年科学基金(11522435)和国家重点研发计划(2017YFA0303103)资助

摘要重费米子超导体是最早发现的非常规超导体, 具有丰富的超导量子现象. 理解重费米子超导配对的微观 起源能够启发非常规超导的机理研究和新型超导体的实验探索。本文简要介绍了近年来在重费米子超导实验和理 论上的最新进展, 利用量子临界自旋涨落的唯象模型, 结合Eliashberg理论计算了 $\mathrm{CeCoIn}$ 的超导性质, 得到了具 有 $\mathrm{d}_{x^{2}-y^{2}}$ 波对称性的超导能隙, 符合实验结果. 在此基础上提出了计算超导转变温度 $T_{\mathrm{c}}$ 的简化公式, 结合二流体理 论解释了 $\mathrm{CeCoIn}$ 和 $\mathrm{CeRhIn}$ 中 $T_{\mathrm{c}}$ 随压力的演化. 这一结果为发展重费米子超导的唯象理论提供了新的思路.

关键词重费米子超导, 二流体理论, Eliashberg 理论, 配对对称性

重费米子材料是一类特殊的强关联电子材料. 在一些包含了f电子元素(如Ce, Yb, U, Pr等镧系或锔 系元素)的金属化合物中, 较为局域的 $\mathrm{f}$ 子自旋(或 局域磁矩) 与导带电子自旋之间存在较强的反铁磁交 换作用(也称Kondo耦合), 在低温下导致相干的自旋 屏蔽效应, 产生巡游的复合准粒子, 其有效质量可以 达到自由电子质量的上百倍. 这类材料被称为重电 子或重费米子材料 ${ }^{[1]}$. 这些由晶格上局域 $\mathrm{f}$ 自旋与导 带电子复合形成的准粒子既具有导带电子的巡游性, 又具有 $\mathrm{f}$ 子的轨道特征, 反映了局域 $\mathrm{f}$ 自旋自由度由 于量子多体效应而产生的巡游重电子行为. $\mathrm{f}$ 电子的 这种局域与巡游的双重属性导致了丰富的量子物态, 如反铁磁、非常规超导、非费米液体等, 以及这些态 之间的共存与竞争.

1979 年, 德国科学家 Steglich等人 ${ }^{[2]}$ 在 $\mathrm{CeCu}_{2} \mathrm{Si}_{2}$ 中观测到了超导电性, 超导转变温度为 $0.5 \mathrm{~K}$, 在 $T_{\mathrm{c}}$ 附近的比热系数 $\gamma \approx 1 \mathrm{~J} /\left(\mathrm{mol} \mathrm{K}^{2}\right)$, 远大于普通金属的
比热系数(如铜约为 $0.7 \mathrm{~mJ} /\left(\mathrm{mol} \mathrm{K} \mathrm{K}^{2}\right)$ ), 表明超导源于 重电子配对; 同时 $T_{\mathrm{c}}$ 、费米温度 $T_{\mathrm{F}}$ 和德拜温度 $\Theta$ 之间 具有近似比例关系 $T_{\mathrm{c}} / T_{\mathrm{F}} \approx T_{\mathrm{F}} / \Theta \approx 0.05$, 与常规超导 体 $\left(T_{\mathrm{F}} \gg \Theta \gg T_{\mathrm{c}}\right)$ 有显著不同, 不能用基于电子-声子 耦合的 Bardeen-Cooper-Schrieffer(BCS) 理论描述 ${ }^{[3]}$, 是一种非常规的超导配对.

重费米子超导是最早发现的非常规超导, 比铜 氧化物高温超导的发现要早 7 年 ${ }^{[4]}$. 迄今为止, 实验 已发现有40多种重费米子超导体, 其能隙结构呈现 多种不同的对称性(s波、p波、d波、f波等)，并且可 能具有不同的超导配对机制 ${ }^{[5]}$. 重费米子的特征能量 尺度较低, 一般只有几个 $\mathrm{meV}$ 量级 ${ }^{[1,6]}$, 因而超导转 变温度也相对较低, 最低只有 $2 \mathrm{mK}\left(\mathrm{YbRh}_{2} \mathrm{Si}_{2}{ }^{[7]}\right)$, 最 高约18.5 K ( $\left.\mathrm{PuCoGa}_{5}{ }^{[8]}\right)$, 但是重电子却表现出较高 的参与度 (即较大的 $T_{\mathrm{c}} / T_{\mathrm{F}}$ ), 这一现象对探索设计新的 高温超导材料可能会有重要的参考价值. 最近十多 年，随着实验技术水平的提高和新型实验手段的应 
用, 对重费米子超导体的实验探索和理论研究都取 得了很多新的进展, 为深人理解非常规超导的微观 机理提供了丰富素材.

\section{1 重费米子超导概况}

超导配对的对称性和微观机理是非常规超导研 究中的重要课题. 不同于一般常规超导体中电子-声 子耦合机制导致的s波配对, 重费米子超导一般具有 非 $\mathrm{s}$ 波的配对对称性, 如 CeCoIn 5 超导具有 $\mathrm{d}_{x^{2}-y^{2}}$ 波对 称性 ${ }^{[9]}$. 大部分重费米子超导的能隙具有点/线节点 (point/line node), 其特征热力学量在 $T_{\mathrm{c}}$ 以下满足幂数 温度依赖行为 ${ }^{[10]}$. 最早发现的重费米子超导体 $\mathrm{CeCu}_{2} \mathrm{Si}_{2}$ 中 $^{[2]}$, 早期测量发现 $T_{\mathrm{c}}$ 以下比热 $C \propto T^{[11,12]}$, 核磁共振自旋晶格弛豫率 $1 / T_{1} \propto T^{3[13]}$, 表明其超导 能隙具有线节点, 符合 $\mathrm{d}$ 波超导的预期. 但是最近有 一些新的实验给出了不同的结论, 如更低温度下的 测量发现, 比热具有指数型温度依赖行为, 同时磁场 下的转角比热系数 $C / T \propto H$, 表明超导是无节点 的 ${ }^{[14]}$, 这一结论在最近的其他实验 ${ }^{[15,16]}$ 中也得到证 实. 类似地, 早期认为存在节点的 $\mathrm{UBe}_{13}$ 在最近的实 验中也受到质疑 ${ }^{[17]}$. 在 $\mathrm{UPt}_{3}$ (B-相超导)和 $\mathrm{URu}_{2} \mathrm{Si}_{2}$ 中, 最新的Kerr效应实验发现, 其超导态破缺了时间反 演对称性 ${ }^{[18,19]}$, 具体机制目前尚不清楚. 这些新的实 验结果要求我们重新认识重费米子超导配对的微观 机理.

从物理上看，重费米子超导具有以下 3 个方面的 特点.

(1) 重费米子超导源自重电子的配对, 因此需要 妥善处理强关联效应及其导致的f电子的局域和巡游 双重属性 (二流体行为). 一些近似的数值方法, 包括 局域密度近似 (local density approximation, LDA) $+X\left(X=\right.$ 库仑相互作用 $\mathrm{U}^{[20,21]}$ 、动力学平均场理论 ${ }^{[22]}$ 、 重整化能带理论 ${ }^{[23]}$ 等) 能够近似给出重电子的能带结 构, 但是无法正确描述 $\mathrm{f}$ 电子的二流体行为及其在外 场调控下的费米面变化, 需要谨慎利用.

（2）重费米子超导往往出现在竞争序附近, 两种 序的共存和竞争会导致许多新奇量子现象, 如 $\mathrm{CeCoIn}_{5}$ 在强磁场下诱导的Q-相具有配对密度波的特 征 ${ }^{[24]}, \mathrm{PrX}_{2} \mathrm{Al}_{20}(\mathrm{X}=\mathrm{Ti}, \mathrm{V})$ 在低温下具有四极矩/八极矩 竞争序行为 ${ }^{[25]}, \mathrm{URu}_{2} \mathrm{Si}_{2}$ 的超导出现在所谓“隐藏序” 内部 ${ }^{[26]}$ 等. 利用掺杂、压力、磁场等手段进行调控, 探索超导态与竞争序之间的关系, 是重费米子超导
研究的一个主要方向 ${ }^{[5]}$

重费米子超导与竞争序的伴生行为, 产生了一 种一般性的认识, 即超导源自竞争序边界的量子临

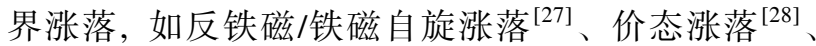
电四极矩涨落 ${ }^{[29]}$ 等, 文献中通常用随机相近似 (random phase approximation, RPA $)^{[21,30]}$ 、涨落交换 (fluctuation-exchange, FLEX)近似 ${ }^{[31]}$ 等方法描述. 本 文将引人量子临界涨落的唯象描述, 用来研究重电 子的超导配对.

(3) f电子的多轨道/多能带特征对重费米子超导 的配对对称性具有重要影响. 对 $\mathrm{CeCu}_{2} \mathrm{Si}_{2}$ 和Pu-115系 列材料 $\left(\mathrm{Pu} M X_{5}, M=\mathrm{Co}, \mathrm{Rh} ; X=\mathrm{Ga}, \mathrm{In}\right)$ 的计算表明，考 虑到多能带效应, 超导可能为具有节点的 $\mathrm{s}$ 波对称 性，而非之前普遍认为的 $\mathrm{d}$ 波对称性 ${ }^{[21,30]}$. 虽然这些 结果还存在争议, 但对理解 $\mathrm{f}$ 电子的轨道特征对重费 米子超导的影响提供了有价值的参考.

总之，由于强关联效应、晶体结构、轨道构型等 多方面的影响, 重费米子超导及其竞争序呈现出丰 富多样的物理，为理解超导的微观特性提供了多元 的视角, 同时亟待理论上的统一认识. 20世纪90年代 Pines课题组 ${ }^{[32 ~ 37]}$ 发展了近反铁磁费米液体唯象理论, 采用唯象的磁化率公式, 很好地解释了铜氧化物超 导体的正常态磁性涨落和超导转变温度等行为. 这 一方法也被应用于重费米子超导的研究，但主要针 对简化的单带格点模型, 难以与实际材料进行比 较 ${ }^{[38]}$. 近年来, 随着实验数据的积累, 开始有人利用 能带计算结合 RPA磁化率研究具体材料中的超导性 质，给出了一些不同以往模型计算的新结果，但目前 仍存在很大争议 ${ }^{[21,30]}$. 我们认为, RPA无法正确描述 重费米子体系中的量子临界涨落，需要能够结合两 种方法的优势: 一方面采用唯象的磁化率描述系统 的磁性涨落(配对力), 更好地处理强关联体系的量子 临界性; 另一方面采用实验提取或电子结构计算得 到的能带，更好地处理实际材料中的能带和费米面 性质. 本文将以 $\mathrm{CeCoIn}_{5}$ 为例, 尝试从唯象的角度建 立重费米子超导的理论模型. 我们得到了和实验一 致的结果, 从原则上支持了上述唯象方法, 为认识重 费米子超导问题提供一个新的可能方案.

\section{2 重费米子超导相图}

为了描述重费米子超导, 必须首先明确产生超 导的强关联电子态的特征. Yang等人 ${ }^{[6,39,40]}$ 整理总结 
了众多材料的普适实验规律, 发展了唯象的重费米 子二流体理论框架, 提出了如图1所示的相图. 在相 干温度 $T^{*}$ 之上, $\mathrm{f}$ 电子表现为近似孤立的局域磁矩, 与 导带电子之间存在较弱的局域自旋耦合或磁性散射; 在 $T^{*}$ 以下, 晶格上的f自旋在Ruderman-Kittel-KasuyaYosida(RKKY) 相互作用下出现较强的磁性关 联 ${ }^{[41 ~ 43]}$, 同时与导带电子发生集体杂化, 部分变得 巡游, 产生了巡游重电子液体 (也称为Kondo液体), 部分仍呈现局域性, 形成自旋液体, 整个体系可以用 两种近似独立的共存流体描述 ${ }^{[6]}$. 在低温下, 若杂化 较弱, 自旋液体占主导, 磁不稳定性有可能导致局域 的磁性长程序, 与少量的Kondo液体共存; 若杂化较 强, $\mathrm{f}$ 电子完全杂化, 会全部形成巡游重电子液体, 产 生重费米液体态; 两种量子态中间会发生量子相变, 强烈的量子临界涨落会诱导巡游重电子配对, 产生 非常规的重费米子超导态.

这一简单图像成功解释了众多重费米子实验中 发现的重电子普适标度行为, 刻画了低温下重费米 子态的温度演化 ${ }^{[44]}$. 在二流体理论中, 超导源自巡 游重电子感受到的局域反铁磁序的量子临界涨落, 两者都起源于 $\mathrm{f}$ 电子, 这种二重性反映了反铁磁自旋 关联与超导之间的复杂关系, 是理解重费米子超导 的关键 ${ }^{[45,46]}$.

\section{$3 \mathrm{CeCoIn}_{5}$ 的强耦合理论计算}

首先以 $\mathrm{CeCoIn}_{5}$ 为例, 基于以上考虑, 结合强耦合 Eliashberg理论, 尝试构建唯象的重费米子超导模型.

量子临界涨落诱导的巡游重电子之间的配对力 可以写为 ${ }^{[35,47]}$

$$
V_{\mathrm{int}}=-g^{2} \chi(\boldsymbol{r}, t) \boldsymbol{s} \cdot \boldsymbol{s}^{\prime},
$$

其中, $g$ 为准粒子和自旋涨落之间的耦合强度, $\chi(\boldsymbol{r}, t)$ 为动力学自旋涨落谱, $s$ 表示巡游重电子的自旋密度.

为了刻画反铁磁量子临界涨落, 利用Millis, Monien和Pines ${ }^{[32]}$ 提出的唯象公式(简称为MMP磁化率)

$$
\chi(\boldsymbol{q}, \omega)=\frac{\chi_{0}}{1+\xi^{2}(\boldsymbol{q}-\boldsymbol{Q})^{2}-\mathrm{i} \omega / \omega_{\mathrm{sf}}},
$$

其中, $\chi_{0}$ 为 $\boldsymbol{Q}$ 处的静态磁化率, $\boldsymbol{Q}, \boldsymbol{\xi}, \omega_{\mathrm{sf}}$ 分别代表反铁 磁涨落的特征波矢、关联长度和特征能标, 其数值可 以从中子散射或核磁共振 (nuclear magnetic resonance, NMR)实验中提取. 这一唯象磁化率公式较好 地描述了铜氧化物和一些重费米子材料中正常态的

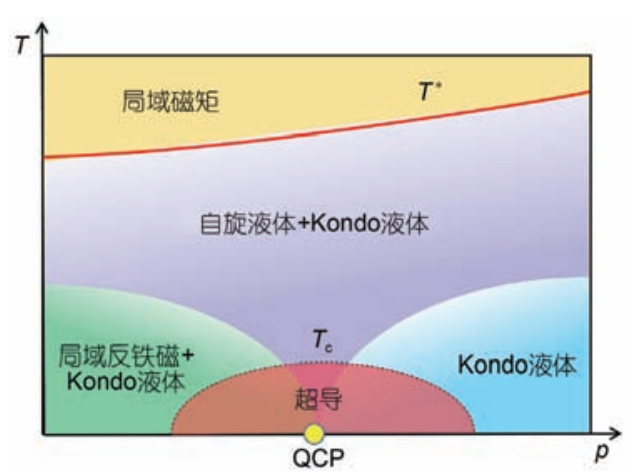

图 1 (网络版彩色)重费米子超导体的二流体相图, 其中QCP表示量 子临界点

Figure 1 (Color online) Schematic plot of the two-fluid phase diagram for heavy fermion superconductors. QCP denotes the quantum critical point

磁性量子临界涨落 ${ }^{[32,38]}$.

在 $\mathrm{CeCoIn}_{5}$ 中, 结合中子散射实验得到的自旋涨 落信息 ${ }^{[48]}$, 可以得到 MMP唯象磁化率的实验参数为: 自旋涨落特征能量 $\omega_{s f} \approx 0.3 \mathrm{meV}$, 反铁磁关联长度 $\xi=9.6 \AA$ (大约为晶格常数 $a=4.62 \AA$ 的 2 倍), 反铁磁波 矢 $\boldsymbol{Q}$ 为 $(\pi, \pi)$.

在Nambu-Gor'kov表象下, 可以推导出超导的 Eliashberg方程组 ${ }^{[38]}$

$$
\begin{aligned}
& \mathrm{i} \omega_{n}\left[1-Z\left(\boldsymbol{k}, \mathrm{i} \omega_{n}\right)\right] \\
& =-\frac{1}{\beta} \sum_{\boldsymbol{k}^{\prime}, \mathrm{i} \omega_{m}} \frac{\mathrm{i} \omega_{m} Z\left(\boldsymbol{k}^{\prime}, \mathrm{i} \omega_{m}\right)}{\omega_{m}^{2} Z^{2}\left(\boldsymbol{k}^{\prime}, \mathrm{i} \omega_{m}\right)+E_{\boldsymbol{k}}^{2}} V_{\boldsymbol{k}, \boldsymbol{k}^{\prime}}\left(\mathrm{i} \omega_{n}-\mathrm{i} \omega_{m}\right), \\
& Z\left(\boldsymbol{k}, \mathrm{i} \omega_{n}\right) \Delta\left(\boldsymbol{k}, \mathrm{i} \omega_{n}\right) \\
& =-\frac{1}{\beta} \sum_{\boldsymbol{k}^{\prime}, \mathrm{i} \omega_{m}} \frac{\Delta\left(\boldsymbol{k}^{\prime}, \mathrm{i} \omega_{m}\right)}{\omega_{m}^{2} Z^{2}\left(\boldsymbol{k}^{\prime}, \mathrm{i} \omega_{m}\right)+E_{\boldsymbol{k}}^{2}} V_{\boldsymbol{k}, \boldsymbol{k}^{\prime}}\left(\mathrm{i} \omega_{n}-\mathrm{i} \omega_{m}\right),
\end{aligned}
$$

其中, $Z\left(\boldsymbol{k}, \mathrm{i} \omega_{n}\right)$ 和 $\Delta\left(\boldsymbol{k}, \mathrm{i} \omega_{n}\right)$ 分别表示准粒子重整化 函数和超导能隙函数

$$
V_{\boldsymbol{k}, \boldsymbol{k}^{\prime}}\left(\mathrm{i} \omega_{n}-\mathrm{i} \omega_{m}\right)=g_{\text {eff }}^{2} \chi\left(\boldsymbol{k}-\boldsymbol{k}^{\prime}, \mathrm{i} \omega_{n}-\mathrm{i} \omega_{m}\right),
$$

其中, $g_{\mathrm{eff}}^{2}=3 g^{2} / 4$ 为磁性量子涨落诱导的自旋单态 的有效配对相互作用, $E_{\boldsymbol{k}}=\sqrt{\xi_{k}^{2}+\Delta_{k}^{2}}$ 为超导态内准 粒子的色散关系, $\xi_{k}$ 为正常态的色散关系.

进而利用扫描隧道显微镜和准粒子干涉技术实验 的数据, 正常态的能带色散关系可用如下公式拟合:

$$
\xi_{k}^{ \pm}=\frac{\varepsilon_{k}^{c}+\varepsilon_{k}^{f}}{2} \pm \sqrt{\left(\frac{\varepsilon_{k}^{c}-\varepsilon_{k}^{f}}{2}\right)^{2}+V_{k}^{2}},
$$

其中, $\varepsilon_{\boldsymbol{k}}^{c}$ 和 $\varepsilon_{\boldsymbol{k}}^{f}$ 分别为导带电子和f电子的色散关系, $V_{k}$ 表示各向异性的杂化函数, 具体表达式及拟合参 
数见文献[9]. 图2给出了两条杂化能带的费米面结构 及费米速度分布的示意图, 可以看出, 图2(a)能带的 费米速度远小于图2(b)能带. 考虑到超导主要源自重 电子配对，为讨论方便，这里只考虑图2(a)中的“重” 电子能带(对应(5)式中 $\xi_{k}=\xi_{k}^{+}$).

基于以上结果, 可以通过数值求解超导对称性 和超导转变温度.

\section{1 超导对称性(弱耦合近似)}

频率依赖性一般对超导能隙结构的动量分布影 响不大，为简单起见，首先考虑弱耦合情形，取

$$
\begin{aligned}
& Z\left(\boldsymbol{k}, \mathrm{i} \omega_{n}\right)=1, \psi_{\boldsymbol{k}} \approx \Delta(\boldsymbol{k}, \mathrm{i} \pi T), \\
& V_{\boldsymbol{k}, \boldsymbol{k}^{\prime}}\left(\mathrm{i} \omega_{n}-\mathrm{i} \omega_{m}\right)=V_{\boldsymbol{k}, \boldsymbol{k}^{\prime}}(0)=V_{\boldsymbol{k}, \boldsymbol{k}^{\prime}} .
\end{aligned}
$$

在 $T \rightarrow T_{\mathrm{c}}$ 时, $\psi_{k} \rightarrow 0, E_{k} \rightarrow\left|\xi_{k}\right|$, 可以对Eliashberg方 程进行线性化处理, 进而对垂直费米面方向作能量 积分, 将动量求和限制在费米面附近, 并对频率进行 求和, 得到如下本征值方程形式

$$
\lambda \psi_{k}=-\oint_{\mathrm{FS}} \frac{\mathrm{d} \boldsymbol{k}_{\|}^{\prime}}{(2 \pi)^{2} v_{\boldsymbol{k}_{\mathrm{F}}}} V_{\boldsymbol{k}, \boldsymbol{k}^{\prime}} \psi_{\boldsymbol{k}^{\prime}},
$$

其中, $v_{k_{\mathrm{F}}}$ 为动量依赖的费米速度, $\lambda$ 为等式右边本征 矩阵的本征值. 在给定相互作用矩阵的情况下, 最大 本征值对应的本征矢量反映了超导能隙的对称性结 构 ${ }^{[49]}$. 在弱耦合近似下, 以上线性化的能隙方程不含
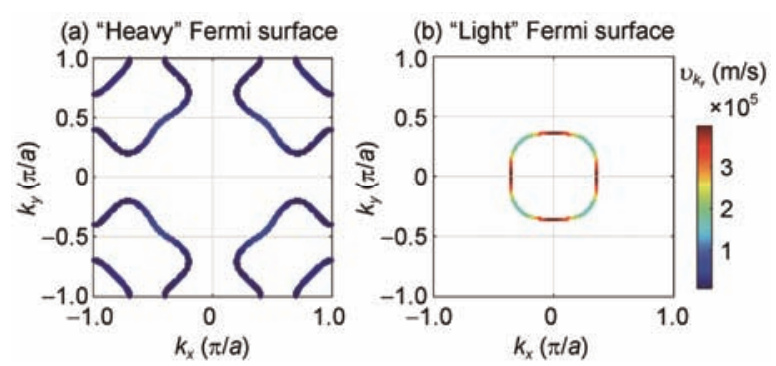

图 2 (网络版彩色) $\mathrm{CeCoIn}_{5}$ 的费米面结构. (a) “重”电子费米面; (b) “轻”电子费米面. $v_{k_{\mathrm{E}}}$ 表示费米速度

Figure 2 (Color online) Fermi surface structures of $\mathrm{CeCoIn}_{5}$. (a) The "heavy" electron Fermi surface; (b) the "light" electron Fermi surface. $v_{\boldsymbol{k}_{\mathrm{F}}}$ represents the Fermi velocity

温度依赖性.

取参数 $g_{\mathrm{eff}}^{2} \chi_{0}=200(\mathrm{meV})^{2}$ 进行计算. 结果表明, 最大本征值对应 $\mathrm{d}_{x^{2}-y^{2}}$ 波, 符合实验测量结果. 图 3(a) (d) 展示了本征值最大的 4 个解对应的超导能隙 在费米面上的分布. 在 $\mathrm{d}_{x^{2}-y^{2}}$ 波情形下, 费米面上通 过 $\boldsymbol{Q}$ 矢量连接的不同区域具有符号相反的 $\psi_{k}$, 可以解 释中子散射实验观测到的自旋共振峰 ${ }^{[48]}$.

进而研究了超导能隙的对称性随化学势 $\left(\xi_{k} \rightarrow \xi_{k}+\delta \mu\right)$ 的变化. 如图 3(e) 所示, 在 -1.27 $\mathrm{meV}<\delta \mu<2 \mathrm{meV}$ 时, $\mathrm{d}_{x^{2}-y^{2}}$ 波 ( $B_{1 \mathrm{~g}}$ 表示)始终占主导; 而当 $\delta \mu<-1.27 \mathrm{meV}$ 时, 各向异性的 $\mathrm{s}$ 波 ( $A_{1 \mathrm{~g}}$ 表示)开始
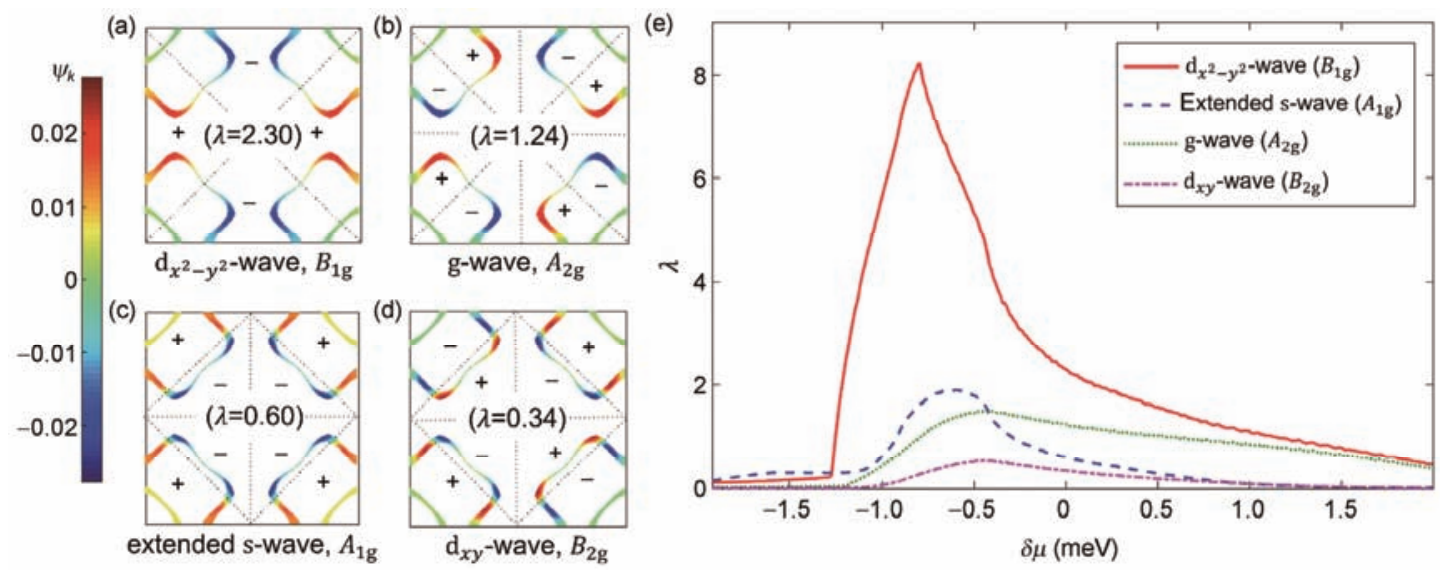

图 3 (网络版彩色)弱耦合近似下CeCoIn 5 超导能隙结构及本征值 $\lambda$ 随化学势的变化. (a) (d) $\delta \mu=0$ 时, 本征值 $\lambda$ 最大的 4 个解对应的超导能隙 结构 $\psi_{k}$ 在费米面上的分布, “+” “-”号反映能隙分布的符号变化; (e) 4 个不同表示下最大 $\lambda$ 解随化学势改变的演化, 其中 $A_{1 \mathrm{~g}}, A_{2 \mathrm{~g}}, B_{1 \mathrm{~g}}, B_{2 \mathrm{~g}}$ 为 $D_{4 h}$ 点 群下的对称性表示

Figure 3 (Color online) Superconducting gap structures of $\mathrm{CeCoIn}_{5}$ and the eigenvalues $\lambda$ as a function of the chemical potential in the weak-coupling calculations. (a)-(d) Gap structures $\left(\psi_{k}\right)$ of the largest 4 eigenvalues at $\delta \mu=0$. The symbol "+" "-" reflect the signs of the gap structure; (e) variation of the largest $\lambda$ as a function of the chemical potential for 4 different symmetry representations $\left(A_{1 \mathrm{~g}}, A_{2 \mathrm{~g}}, B_{1 \mathrm{~g}}, B_{2 \mathrm{~g}}\right)$ of the point group $D_{4 h}$ 
占主导. $\mathrm{d}_{x^{2}-y^{2}}$ 波解在 $\delta \mu<-1.27 \mathrm{meV}$ 时存在明显的转 折, 过渡到同属 $B_{1 \mathrm{~g}}$ 表示的另一本征解. 在 $\delta \mu<-0.79$ $m e V$ 附近, $\mathrm{d}_{x^{2}-y^{2}}$ 波表现出尖锐的峰值结构, 这是由 于费米面拓扑结构在此处发生突变(Lifshitz相变). 在 $\delta \mu$ 接近 $2 \mathrm{meV}$ 时, $\mathrm{d}_{x^{2}-y^{2}}$ 波和 $g$ 波近似简并.

\section{2 超导转变温度 $T_{\mathrm{c}}$ (强耦合近似)}

基于弱耦合情形得到的 $\mathrm{d}_{x^{2}-y^{2}}$ 波能隙的动量分布 结构, 可以进一步约化强耦合框架下的线性化 Eliashberg方程组, 对超导转变温度进行更精细的计 算. 假设 $\Delta\left(\boldsymbol{k}, \mathrm{i} \omega_{n}\right)=\psi_{\boldsymbol{k}} \tilde{\Delta}\left(\mathrm{i} \omega_{n}\right)$, 为了保证本征矩阵的 对称性(或厄米性), 在方程 (4)两边同时乘上归一化 的 $\psi_{k}$, 然后积分掉动量 (只考虑费米面及其附近的 点), 可以得到如下频率依赖的本征能隙方程

$$
\sum_{\mathrm{i} \omega_{m}}\left[K\left(\mathrm{i} \omega_{n}-\mathrm{i} \omega_{m}\right)-\frac{\tilde{Z}\left(\mathrm{i} \omega_{n}\right)\left|\omega_{n}\right|}{\pi T} \delta_{m, n}\right] \frac{\tilde{\Delta}\left(\mathrm{i} \omega_{m}\right)}{\left|\omega_{m}\right|}=0,
$$

其中,

$$
\begin{gathered}
\tilde{Z}\left(\mathrm{i} \omega_{n}\right)=\oint_{\mathrm{FS}} \frac{\mathrm{d} \boldsymbol{k}_{\|}}{(2 \pi)^{2} v_{\boldsymbol{k}_{\mathrm{F}}}} Z\left(\boldsymbol{k}, \mathrm{i} \omega_{n}\right) \psi_{\boldsymbol{k}}^{2} / \oint_{\mathrm{F} S} \frac{\mathrm{d} \boldsymbol{k}_{\|}}{(2 \pi)^{2} v_{\boldsymbol{k}_{\mathrm{F}}}} \psi_{\boldsymbol{k}}^{2}, \\
K\left(\mathrm{i} \omega_{n}-\mathrm{i} \omega_{m}\right) \\
=\frac{\oint_{\mathrm{FS}} \frac{\mathrm{d} \boldsymbol{k}_{\|}}{(2 \pi)^{2} v_{\boldsymbol{k}_{\mathrm{F}}}} \oint_{\mathrm{FS}} \frac{\mathrm{d} \boldsymbol{k}_{\|}^{\prime}}{(2 \pi)^{2} v_{\boldsymbol{k}_{\mathrm{F}}}} V_{\boldsymbol{k}, \boldsymbol{k}^{\prime}}\left(\mathrm{i} \omega_{n}-\mathrm{i} \omega_{m}\right) \psi_{\boldsymbol{k}} \psi_{\boldsymbol{k}}^{\prime}}{\oint_{\mathrm{FS}} \frac{\mathrm{d} \boldsymbol{k}_{\|}}{(2 \pi)^{2} v_{\boldsymbol{k}_{\mathrm{F}}}} \psi_{k}^{2}} .
\end{gathered}
$$

其中, 分母上的 $\oint_{\mathrm{F} S} \frac{\mathrm{d} \boldsymbol{k}_{\|}}{(2 \pi)^{2} v_{\boldsymbol{k}_{\mathrm{F}}}} \psi_{k}^{2}$ 是为了消除 $\psi_{k}$ 的量纲 的影响. 通过对角化 (7)式中的本征矩阵, 可以求解 本征值 $\lambda^{\prime}$ 随温度的演化, 当最大本征值演化到 0 时即 对应超导转变温度 $T_{\mathrm{c}}$.

图4给出了 $\lambda^{\prime}$ 随温度演化的计算结果. 从图4(a) 可以看到, 各个本征值随温度的演化, 在高温时都趋 于负常数. 最大本征值 $\lambda_{\max }^{\prime}$ 具有明显的温度依赖性, 在 $T \rightarrow 0$ 时趋于发散. 图4(b)给出了不同 $g_{\text {eff }}^{2} \chi_{0}$ 值下 $\lambda_{\text {max }}^{\prime}$ 随温度变化的曲线. 可以看到, 随着 $g_{\text {eff }}^{2} \chi_{0}$ 值的 增大, $\lambda_{\text {max }}^{\prime}(T)$ 的零点沿横轴方向右移, 反映出超导 转变温度 $T_{\mathrm{c}}$ 的升高.

进一步分析了 $\omega_{s f}$ 和 $g_{\text {eff }}^{2} \chi_{0}$ 对 $T_{\mathrm{c}}$ 的影响, 结果如 图5(a)和(b)所示. 在不同 $g_{\text {eff }}^{2} \chi_{0}$ 取值下, $T_{\mathrm{c}}$ 随 $\omega_{\mathrm{sf}}$ 表现 出线性递增行为. 而在固定 $\omega_{s f}$ 时, $T_{\mathrm{c}}$ 随 $g_{\text {eff }}^{2} \chi_{0}$ 呈现非
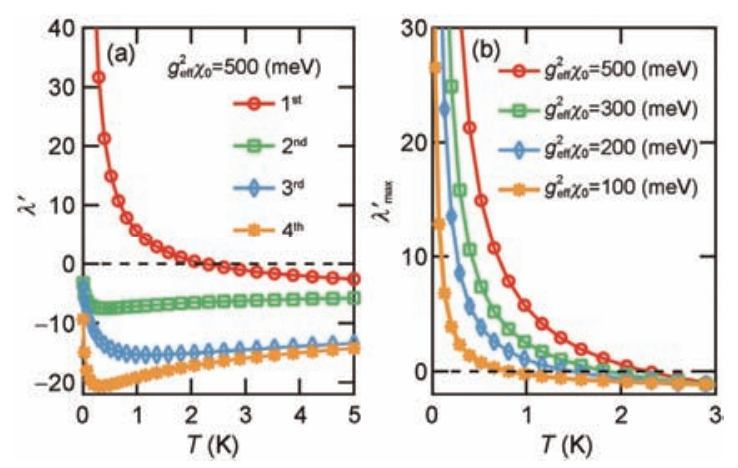

图 4 (网络版彩色)强耦合近似下本征值随温度的演化. (a) $g_{\text {eff }}^{2} \chi_{0}=500 \mathrm{meV}$ 时, $\lambda^{\prime}$ 最大的 4 个解随 $T$ 的演化; (b) 最大本征值 $\lambda_{\max }^{\prime}$ 在不同 $g_{\text {eff }}^{2} \chi_{0}$ 取值下随温度的演化. 实线代表 $\lambda^{\prime}=0$

Figure 4 (Color online) Temperature evolution of the eigenvalues in the strong-coupling calculations. (a) $T$-evolution of the 4 leading solutions of $\lambda^{\prime}$ at $g_{\text {eff }}^{2} \chi_{0}=500 \mathrm{meV}$; (b) $T$-evolution of the largest eigenvalue $\lambda_{\max }^{\prime}$ at different $g_{\text {eff }}^{2} \chi_{0}$. The solid lines denote $\lambda^{\prime}=0$

线性增长的依赖关系. 如图 5(c) 所示, 参考对铜氧化 物超导的类似研究 ${ }^{[00]}$, 发现 $T_{\mathrm{c}}$ 具有如下表达式:

$$
T_{\mathrm{c}}=\alpha T_{\mathrm{sf}} \exp \left(-1 / \beta g_{\text {eff }}{ }^{\prime} N_{\mathrm{F}}\right),
$$

其中, $\alpha, \beta$ 是拟合常数, $T_{\mathrm{sf}}=\omega_{\mathrm{sf}}(\xi / a)^{2}$ 为自旋涨落特 征温度, $N_{\mathrm{F}}$ 为费米能处的态密度. 这一结果表明, $T_{\mathrm{c}}$ 正比于 $T_{\mathrm{sf}}$, 与文献[8]的支撑材料中给出的许多非常 规超导体的 $T_{\mathrm{sf}}-T_{\mathrm{c}}$ 线性关系一致.

\section{3 实验对比}

以上理论推导原则上只在费米液体区成立. 为 了与实验对比, 将其进行唯象的推广, 应用于所有存 在重电子的区域，包括非费米液体区和小费米面的 反铁磁区 (如 CeRhIn $_{5}$ ). 同时考虑到特征温度 $T^{*}$ 源自 $\mathrm{f}$ 电子之间的RKKY相互作用, 既是决定巡游重电子的 基本能标, 也是决定反铁磁自旋涨落的基本能量尺 度, 控制着超导转变温度的大小 ${ }^{[45,46]}$. 因此假设 $g_{\mathrm{eff}}^{\prime}$ 由 $T^{*}$ 决定, 而 $T_{s f}$ 不随压力变化, 得到一个简单的类似 $\mathrm{BCS}$ 的 $T_{\mathrm{c}}$ 公式 ${ }^{[45,46]}$

$$
\begin{aligned}
T_{\mathrm{c}}(p) & =0.14 T_{m}^{*} \exp \left[-\frac{1}{N_{\mathrm{F}}\left(p, T_{\mathrm{c}}\right) V_{\text {eff }}(p)}\right] \\
& =0.14 T_{m}^{*} \exp \left[-\frac{1}{\eta \kappa\left(p, T_{\mathrm{c}}\right)}\right],
\end{aligned}
$$

其中, $N_{\mathrm{F}}\left(p, T_{\mathrm{c}}\right)$ 是 $T_{\mathrm{c}}$ 处的重电子态密度, 可以由二流体理 论的预言得到, $V_{\mathrm{eff}}(p)=\eta k_{\mathrm{B}} T^{*}(p)$ 为有效配对相互作 

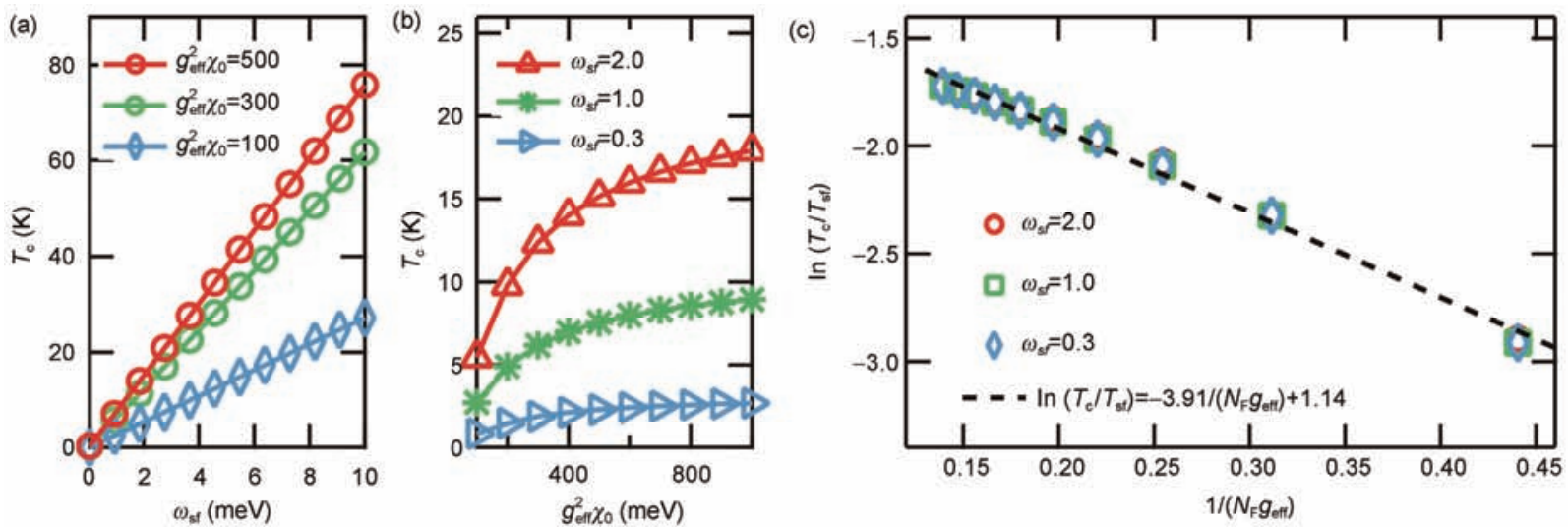

图 5 (网络版彩色) $T_{\mathrm{c}}$ 对 $g_{\mathrm{eff}}^{2} \chi_{0}$ 和 $\omega_{s f}$ 的依赖关系. (a) 在不同 $g_{\mathrm{eff}}^{2} \chi_{0}$ 值下 $T_{\mathrm{c}}$ 随 $\omega_{\mathrm{sf}}$ 的变化曲线; (b) 在不同 $\omega_{\mathrm{sf}}$ 下 $T_{\mathrm{c}}$ 随 $g_{\text {eff }}^{2} \chi_{0}$ 的变化曲线; (c) $T_{\mathrm{c}}$ 随 $N_{\mathrm{F}} g_{\mathrm{eff}}$ 的标度关系

Figure 5 (Color online) Dependence of $T_{\mathrm{c}}$ on $g_{\text {eff }}^{2} \chi_{0}$ and $\omega_{s f}$. (a) $T_{\mathrm{c}}$ as a function of $\omega_{\text {sf }}$ for different $g_{\text {eff }}^{2} \chi_{0}$; (b) $T_{\mathrm{c}}$ as a function of $g_{\text {eff }}^{2} \chi_{0}$ for different $\omega_{s f} ;(\mathrm{c})$ a scaling relation of $T_{\mathrm{c}}$ versus of $N_{\mathrm{F}} g_{\text {eff }}$

用, $\kappa\left(p, T_{\mathrm{c}}\right)=N_{\mathrm{F}}\left(p, T_{\mathrm{c}}\right) k_{\mathrm{B}} T^{*}(p)$ 是无量纲的特征堣合强 度, $\eta$ 是材料相关的拟合系数, $T_{m}^{*}$ 为最大 $T_{\mathrm{c}}$ 时的相干温 度, 可以通过实验测到, 系数 0.14 也源于实验拟合 ${ }^{[46]}$.

在实际拟合中, $T^{*}(p)$ 可以通过实验测量得到, 重电子态密度具有如下普适温度依赖性 ${ }^{[39,40]}$ :

$$
N_{\mathrm{F}}(T) \propto f_{0}\left(1-\left(T / T^{*}\right)\right)^{3 / 2}\left(1+\ln \left(T^{*} / T\right)\right),
$$

其中, $f_{0}$ 刻画 $\mathrm{f}$ 电子与导带电子发生集体杂化的效率, 其数值随压强变化, 拟合时假设近似正比于 $T^{*}(p)$. 为了计算 $T_{\mathrm{c}}(p)$ 处的重电子态密度, 需要将以上对数 发散截断. 如图6所示, (1) 当 $T_{\mathrm{c}}<T_{\mathrm{L}}$ 时, $\mathrm{f}$ 电子在形成
超导时已经完全退局域化, $N_{\mathrm{F}}(T)$ 在退局域化温度 $T_{\mathrm{L}}$ 处截断; (2) 当 $T_{\mathrm{c}}<T_{\mathrm{N}}$ 时, 由于磁性长程序的出现, f电 子在低温下可能发生重新局域化行为, 可以近似取 $T_{\mathrm{N}}$ 或长程反铁磁关联出现的温度 $T_{0}$ 为截断温度; $(3)$ 在中间区, $T_{\mathrm{c}}>T_{\mathrm{N}}$ 且 $T_{\mathrm{c}}>T_{\mathrm{L}}$, 取 $T_{\mathrm{c}}$ 本身为截断温度, 通 过自洽求解得到态密度 $N_{\mathrm{F}}\left(p, T_{\mathrm{c}}\right)$. 具体计算细节参 见文献[46].

图6给出了对 $\mathrm{CeCoIn}_{5}$ 和 $\mathrm{CeRhIn}$ 的压力-温度相 图的 $T_{\mathrm{c}}$ 拟合结果，由以上公式预言的 $T_{\mathrm{c}}$ 理论值与实验 值能很好地符合. 其中 $T_{\mathrm{c}}$ 随压力的非单调行为源自 两种不同的效应：在低压区，重电子随压力降低更加
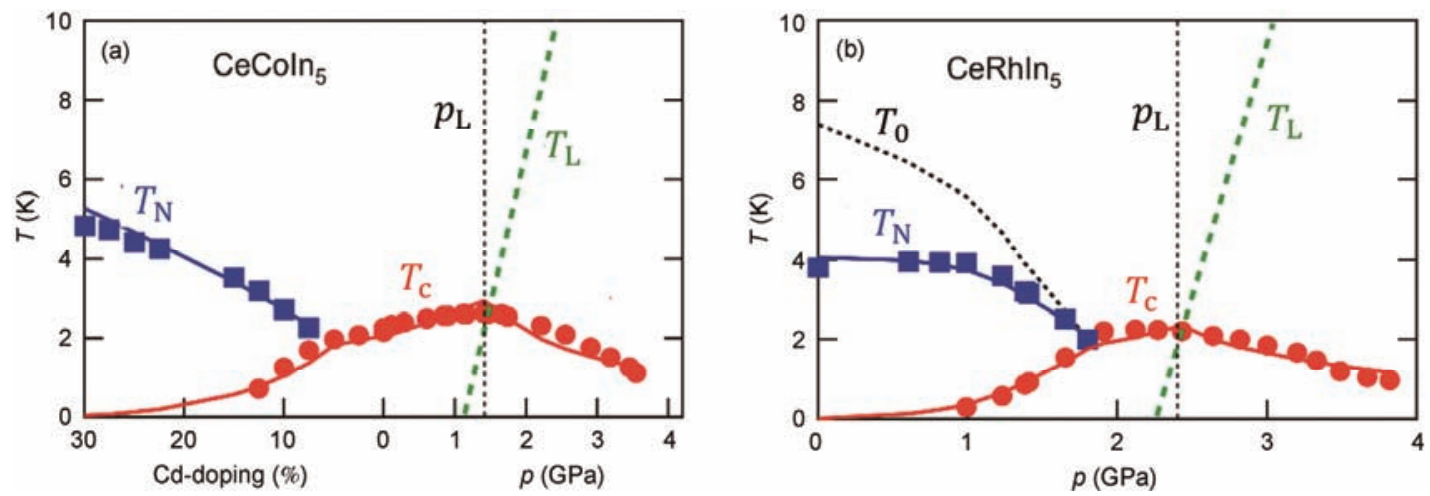

图 6 (网络版彩色) 对 $T_{\mathrm{c}}$ 随压力和掺杂变化的实验拟合. (a) $\mathrm{CeCoIn}_{5}$; (b) $\mathrm{CeRhIn}$. 其中实线为理论预测值, 圆点标记为 $T_{\mathrm{c}}$ 的实验值, 方形标记为 反铁磁转变温度 $T_{\mathrm{N}}$ 的实验值, $T_{0}$ 为实验测得的长程反铁磁关联出现的温度, $T_{\mathrm{L}}$ 为二流体模型计算给出的 $\mathrm{f}$ 子完全退局域化的温度, $p_{\mathrm{L}}$ 为 $T_{\mathrm{c}}$ 与 $T_{\mathrm{L}}$ 相等时的压强. 关于拟合的具体细节可参考文献[46]

Figure 6 (Color online) Comparison of experimental measured $T_{\mathrm{c}}$ as a function of pressure or doping. (a) CeCoIn 5 ; (b) CeRhIn 5 . The solid lines denote the theoretical predictions, the points are the experimental data for $T_{\mathrm{c}}$ (circles) and the Néel temperature $T_{\mathrm{N}}$ (squares), $T_{0}$ marks the measured onset of the long-range antiferromagnetic correlations, $T_{\mathrm{L}}$ denotes the predicted temperature below which all $\mathrm{f}$ electrons become fully delocalized, and $p_{\mathrm{L}}$ is the pressure where $T_{\mathrm{c}}$ and $T_{\mathrm{L}}$ intersect ${ }^{[46]}$ 
局域化, 导致费米面附近的态密度降低, 抑制超导; 而在高压区, 重电子随压力增加更加巡游, 带宽增 加, 同样导致态密度降低, 抑制超导; 从而在量子临 界点附近出现最大 $T_{\mathrm{c}}$. 这一结果表明局域性和过度 的巡游性均不利于超导配对.

\section{4 结论与展望}

简而言之, 利用强耦合的Eliashberg方程研究了 重费米子材料 $\mathrm{CeCoIn}$ 的超导性质, 基于自旋涨落机 制成功解释了其超导能隙的 $\mathrm{d}$ 波对称性, 提出了超导 转变温度 $T_{\mathrm{c}}$ 的简单公式, 并结合重费米子二流体模 型解释了 $T_{\mathrm{c}}$ 随压力的 “拱顶”行为. 这些结果从原则 上支持了我们所采用的近似方法. 我们还将其推广 到多带或多轨道系统, 研究了重费米子材料 $\mathrm{CeCu}_{2} \mathrm{Si}_{2}$ 的超导性质, 发现带间跃迁对超导能隙对称性具有 重要影响, 此工作正在进行中. 结合前面提到过的重
费米子超导研究的主要困难, 我们计划在未来的工 作中进一步完善这一唯象理论框架, 更好地处理f电 子的轨道特征、多种不同类型的量子临界涨落、以及 $\mathrm{f}$ 电子的局域-巡游二重性, 扩展其应用范围, 并对更 多的超导态性质进行理论研究和实验对照, 为深人 认识重费米子超导提供一个唯象的理论基础.

近十多年来, 许多实验技术, 如扫描隧道显微

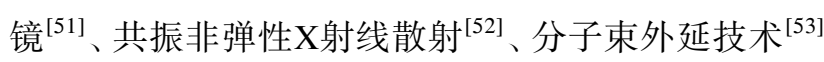
等, 开始应用于重费米子超导研究, 加深了人们对重 费米子超导的微观物理认识. 一些实验上新发现的 奇异量子现象, 如 $\mathrm{CeCoIn}$ 虫超导与磁性共存的 $\mathrm{Q}$ 相 ${ }^{[54]} 、 \mathrm{YbRh}_{2} \mathrm{Si}_{2}{ }^{[7]}$ 和Pu-115体系 ${ }^{[55]}$ 中超导的配对机制, 以及 $\mathrm{CeCu}_{2} \mathrm{Si}_{2}{ }^{[14]} 、 \mathrm{UBe}_{13}{ }^{[17]}$ 等体系的超导能隙对称性 等, 都亟待理论上给出合理的描述. 对重费米子超导 微观机理的研究, 可能会对探索具有更高转变温度 的新型超导材料产生重要启发.

\section{参考文献}

White B D, Thompson J D, Maple M B. Unconventional superconductivity in heavy-fermion compounds. Phys C, 2015, 514: 246-278

Steglich F, Aarts J, Bredl C D, et al. Superconductivity in the presence of strong Pauli paramagnetism: $\mathrm{CeCu}_{2} \mathrm{Si}_{2}$. Phys Rev Lett, 1979, 43: 1892-1896

Bardeen J, Cooper L N, Schrieffer J R. Theory of superconductivity. Phys Rev, 1957, 108: 1175-1204

Bednorz J G, Müller K A. Possible high $T_{\mathrm{c}}$ superconductivity in the Ba-La-Cu-O system. Zeitschr Phys B, 1986, 64: 189-193

Yang Y F, Li Y. Heavy-fermion superconductivity and competing orders (in Chinese). Acta Phys Sin, 2015, 64: 217401 [杨义峰, 李宇. 重费米子超导与竞争序. 物理学报, 2015, 64: 217401]

Yang Y F, Fisk Z, Lee H O, et al. Scaling the Kondo lattice. Nature, 2008, 454: 611-613

Schuberth E, Tippmann M, Steinke L, et al. Emergence of superconductivity in the canonical heavy-electron metal $\mathrm{YbRh}_{2} \mathrm{Si}_{2} . \mathrm{Science}$ 2016, 351: 485-488

Curro N J, Caldwell T, Bauer E D, et al. Unconventional superconductivity in PuCoGa5. Nature, 2005, 434: 622-625

Allan M P, Massee F, Morr D K, et al. Imaging Cooper pairing of heavy fermions in CeCoIn 5 . Nat Phys, 2013, 9: 468-473

Sigrist M, Ueda K I. Phenomenological theory of unconventional superconductivity. Rev Mod Phys, 1991, 63: 239-311

Bredl C D, Spille H, Rauchschwalbe U, et al. Gapless superconductivity and variation of $T_{\mathrm{c}}$ in the heavy-fermion system $\mathrm{CeCu}_{2} \mathrm{Si}_{2} . \mathrm{J}$ Magn Magn Mater, 1983, 31: 373-376

Bredl C D, Lieke W, Schefzyk R, et al. Specific heat and thermal expansion of $\mathrm{CeCu}_{2} \mathrm{Si}_{2}$ at low temperature. J Magn Magn Mater, 1985, 47: $30-32$

Kitaoka Y, Ueda K I, Fujiwara K, et al. NMR investigation of superconductivity and Kondo-coherency in $\mathrm{CeCu}_{2} \mathrm{Si}_{2}$. J Phys $\mathrm{Soc} \mathrm{Jpn}_{\text {, }}$ 1986, 55: 723-726

Kittaka S, Aoki Y, Shimura Y, et al. Multiband superconductivity with unexpected deficiency of nodal quasiparticles in $\mathrm{CeCu}_{2} \mathrm{Si}_{2}$. $\mathrm{Phys}$ Rev Lett, 2014, 112: 067002

Pang G M, Smidman M, Zhang J L, et al. Evidence for fully gapped d-wave superconductivity in $\mathrm{CeCu}_{2} \mathrm{Si}_{2}$. arXiv: 1605.04786v1

Yamashita T, Takenaka T, Tokiwa Y, et al. Fully gapped superconductivity with no sign change in the prototypical heavy-fermion $\mathrm{CeCu}_{2} \mathrm{Si}_{2}$. Sci Adv, 2017, 3: e1601667

Shimizu Y, Kittaka S, Sakakibara T, et al. Field-orientation dependence of low-energy quasiparticle excitations in the heavy-electron superconductor $\mathrm{UBe}_{13}$. Phys Rev Lett, 2015, 114: 147002

Schemm E R, Gannon W J, Wishne C M, et al. Observation of broken time-reversal symmetry in the heavy-fermion superconductor UPt ${ }_{3}$. Science, 2014, 345: 190-193 
19 Schemm E R, Baumbach R E, Tobash P H, et al. Evidence for broken time-reversal symmetry in the superconducting phase of URu $\mathrm{Si}_{2}$. Phys Rev B, 2015, 91: 140506

Suzuki M T, Harima H. Change of Fermi surface topology in $\mathrm{CeRu}_{2} \mathrm{Si}_{2}$ studied by LSDA+U method. J Phys Soc Jpn, 2010, 79: 024705

Ikeda H, Suzuki M T, Arita R. Emergent loop-nodal $\mathrm{s}_{ \pm}$-wave superconductivity in $\mathrm{CeCu}_{2} \mathrm{Si}_{2}$ : Similarities to the iron-based superconductors. Phys Rev Lett, 2015, 114: 147003

Shim J H, Haule K, Kotliar G. Modeling the localized-to-itinerant electronic transition in the heavy fermion system CeIrIn 5 . Science, 2007, 318: $1615-1617$

Zwicknagl G. The utility of band theory in strongly correlated electron systems. Rep Prog Phys, 2016, 79: 124501

Gerber S, Bartkowiak M, Gavilano J L, et al. Switching of magnetic domains reveals spatially inhomogeneous superconductivity. Nat Phys, 2014, 10: 126-129

Tsujimoto M, Matsumoto Y, Tomita T, et al. Heavy-fermion superconductivity in the quadrupole ordered state of $\operatorname{PrV}_{2} \mathrm{Al}_{20}$. Phys Rev Lett, 2014, 113: 267001

Kung H H, Baumbach R E, Bauer E D, et al. Chirality density wave of the "hidden order" phase in URu $\mathrm{Si}_{2}$. Science, 2015, 347: 1339-1342

Scalapino D J. A common thread: The pairing interaction for unconventional superconductors. Rev Mod Phys, 2012, 84: 1383-1417

Miyake K, Watanabe S. Unconventional quantum criticality due to critical valence transition. J Phys Soc Jpn, 2014, 83: 061006

Thalmeier P, Maki K, Yuan Q. Multiple superconducting phases in skutterudite PrOs $\mathrm{Sb}_{12}$. Phys C, 2004, 408-410: 177-178

Das T, Zhu J X, Graf M J. Theory of nodal s-wave pairing symmetry in the Pu-based 115 superconductor family. Sci Rep, 2015, 5: 8632

Takimoto T, Hotta T, Ueda K I. Strong-coupling theory of superconductivity in a degenerate Hubbard model J. Phys Rev B, 2004, 69: 104504

Millis A J, Monien H, Pines D. Phenomenological model of nuclear relaxation in the normal state of $\mathrm{YBa}_{2} \mathrm{Cu}_{3} \mathrm{O}_{7}$. Phys $\mathrm{Rev} \mathrm{B}$, 1990, 42: 167-178

Monthoux P, Balatsky A V, Pines D. Toward a theory of high-temperature superconductivity in the antiferromagneticaliy correlated Cuprate oxides. Phys Rev Lett, 1991, 67: 3448-3451

Monthoux P, Balatsky A V, Pines D. Weak-coupling theory of high-temperature superconductivity in the antiferromagnetically correlated copper oxides. Phys Rev B, 1992, 46: 14803-14817

Monthoux P, Lonzarich G G. p-wave and d-wave superconductivity in quasi-two-dimensional metals. Phys Rev B, 1999, 59: 14598-14605

Monthoux P, Lonzarich G G. Magnetically mediated superconductivity in quasi-two and three dimensions. Phys Rev B, 2001, 63: 054529

Monthoux P, Lonzarich G G. Magnetically mediated superconductivity: Crossover from cubic to tetragonal lattice. Phys Rev B, 2002, 66: 224504

Nishiyama S, Miyake K, Varma C M. Superconducting transition temperatures for spin-fluctuation superconductivity: Application to heavy-fermion compounds. Phys Rev B, 2013, 88: 014510

Yang Y F, Pines D. Universal behavior in heavy-electron materials. Phys Rev Lett, 2008, 100: 096404

Yang Y F, Pines D. Emergent states in heavy-electron materials. Proc Natl Acad Sci USA, 2012, 109: E3060-E3066

Ruderman M A, Kittel C. Indirect exchange coupling of nuclear magnetic moments by conduction electrons. Phys Rev, 1954, 96: 99-102

Kasuya T. A theory of metallic ferro- and antiferromagnetism on Zener's model. Prog Theor Phys, 1956, 16: 45-57

Yosida K. Magnetic properties of Cu-Mn alloys. Phys Rev, 1957, 106: 893-898

Yang Y F. Two-fluid model for heavy electron physics. Rep Prog Phys, 2016, 79: 074501

Yang Y F, Pines D, Curro N J. Quantum critical scaling and superconductivity in heavy electron materials. Phys Rev B, 2015, 92 : 195131

Yang Y F, Pines D. Emergence of superconductivity in heavy-electron materials. Proc Natl Acad Sci USA, 2014, 111: 18178-18182

Monthoux P, Pines D, Lonzarich G G. Superconductivity without phonons. Nature, 2007, 450: 1177-1183

Stock C, Broholm C, Hudis J, et al. Spin resonance in the d-wave superconductor CeCoIn 5 . Phys Rev Lett, 2008, 100: 087001

Graser S, Maier T A, Hirschfeld P J, et al. Near-degeneracy of several pairing channels in multiorbital models for the Fe pnictides. New J Phys, 2009, 11: 025016

Monthoux P, Pines D. Spin-fluctuation-induced superconductivity in the copper oxides: A strong coupling calculation. Phys Rev Lett, 1992, 69: 961-964

Aynajian P, da Silva Neto E H, Gyenis A, et al. Visualizing heavy fermions emerging in a quantum critical Kondo lattice. Nature, 2012, 486: 201-206

Wray L A, Denlinger J, Huang S W, et al. Spectroscopic determination of the atomic f-electron symmetry underlying hidden order in $\mathrm{URu}_{2} \mathrm{Si}_{2}$. Phys Rev Lett, 2015, 114: 236401

Shishido H, Shibauchi T, Yasu K, et al. Tuning the dimensionality of the heavy fermion compound CeIn 3 . Science, 2010, 327: 980-983

Kim D Y, Lin S Z, Weickert F, et al. Intertwined orders in heavy-fermion superconductor CeCoIn 5 . Phys Rev X, 2016, 6: 041059

Bauer E D, Thompson J D. Plutonium-based heavy-fermion systems. Annu Rev Condens Matter Phys, 2015, 6: 137-153 


\title{
A phenomenological theory of heavy fermion superconductivity in $\mathrm{CeCoIn}_{5}$
}

\author{
LI Yu ${ }^{1,2} \&$ YANG YiFeng ${ }^{1,2,3^{*}}$ \\ ${ }^{1}$ Beijing National Laboratory for Condensed Matter Physics, Institute of Physics, Chinese Academy of Sciences, Beijing 100190, China; \\ ${ }^{2}$ University of Chinese Academy of Sciences, Beijing 100049, China; \\ ${ }^{3}$ Collaborative Innovation Center of Quantum Matter, Beijing 100190, China \\ * Corresponding author, E-mail: yifeng@iphy.ac.cn
}

Unconventional superconductivity was first discovered in heavy fermion materials which exhibit a rich variety of superconducting quantum phenomena. Understanding the microscopic origin of heavy fermion superconductivity will help us understand the nature of high-temperature superconductivity and explore new class of unconventional superconductors. In this article, we give a brief introduction to the recent theoretical and experimental studies on heavy fermion superconductors. In particular, it has been shown that previous understandings of the pairing mechanism based on oversimplified single-band model calculations may not explain the recent experimental observations of the superconducting gap symmetry and therefore need to be revisited. For example, the heavy fermion superconductors $\mathrm{CeCu}_{2} \mathrm{Si}_{2}$ and $\mathrm{UBe}_{13}$, which have long been believed to have nodal superconducting gap structures for over three decades, are now found to exhibit nodeless behaviors in many new experiments. While these may be partially explained by using realistic band structures in combination with random phase approximation (RPA) for the dynamic susceptibility, we point out that for strongly correlated systems such as heavy fermions, RPA fails to capture the true behavior of quantum critical fluctuations which act as the pairing force for the unconventional superconductivity. We argue that there are three major issues that need to be taken into account in order to develop a good understanding of the heavy fermion superconductivity: (1) the strong electronic correlations and the two-fluid behavior of the f electrons; (2) the quantum critical nature of the superconducting pairing force that cannot be obtained based on RPA; (3) the multi-band or multi-orbital properties that rely on real materials and may be crucial for the gap structures. Following these considerations, we propose a new framework based on the strong-coupling Eliashberg theory that combines previous phenomenological theory of the spin-fluctuation-induced pairing mechanism and realistic band structures from either experimental measurements or first-principles calculations. As an example, we apply our model to the prototype heavy fermion superconductors $\mathrm{CeCoIn}_{5}$ and $\mathrm{CeRhIn}_{5}$. By using a single-band model derived from the scanning tunneling spectroscopy, we solve the linearized Eliashberg equation and produce the correct d-wave superconducting gap structure, in agreement with experimental observations. We further predict a simple formula for the superconducting transition temperature $T_{\mathrm{c}}$ as a function of the pairing strength and the spin fluctuation energy. We then extend the formula to general cases and use the two-fluid prediction on the heavy electron density of states to calculate the pressure-variation of $T_{\mathrm{c}}$. Our results agree well with experiment and explain the dome structure of $T_{\mathrm{c}}$. For multi-band systems, we have studied the superconductivity in $\mathrm{CeCu}_{2} \mathrm{Si}_{2}$. In contrast to previous calculations that predict either d-wave or nodal s-wave gap, we found that the inter-band scattering plays an essential role and may cause a nodeless gap structure. This work is still under progress. We believe that the success of the new framework suggests that it may provide a promising basis for treating the above issues and will help our understanding of the properties of heavy fermion superconductivity. In the future, we hope to extend our study to other heavy fermion superconductors and take into consideration the detailed orbital characters and the dual nature of $f$ electrons. The latter would possibly require a reformulation of the Eliashberg equations.

heavy fermion superconductivity, two-fluid theory, Eliashberg theory, pairing symmetry

doi: 10.1360/N972017-00698 